\title{
Binge Eating among Women Who Suffer from Psychological Abuse in Their Relationship: The Moderating Role of Defense Mechanisms
}

\author{
${ }^{1}$ Orly Yona Drori, ${ }^{2}$ Shirley Ben Shlomo \\ ${ }^{1}$ MSW, Clinical Dietitian B.Sc. Lev Hasharon Mental Health Medical Center. \\ Pardessyia; Israel, Ph.D. , ${ }^{2}$ The Louis and Gabi Weisfeld School of Social Work. Bar Ilan \\ University, Ramat Gan; Israel
}

\begin{abstract}
.
Objectives: The participants will be able to recognize the predictors of Binge Eating addiction among women who suffer from psychological abuse in their relationships and will recognize the role of woman's defense mechanisms in moderating the association between psychological abuse and Binge Eating addiction.

Methods: A convenience sample of 380 Israeli women in relationships were located via the Internet, and after consenting to participate in the study, they completed a series of structured questionnaires (The Yale Food Addiction Scale; The Defense Style Questionnaire; Psychological maltreatment of women by their male partners; Level of Differentiation of Self; sociodemographic questionnaire).

Results: The higher the level of differentiation and mature defense mechanisms, the less addictive a woman is. However, the level of addiction among women who experience psychological abuse with in their intimate relations, is higher than women who do not experience psychological abuse in their relationship. Among women who experienced psychological abuse in their relations, the defense mechanisms moderate the association between psychological abuse within intimate relations and the extent of the addiction to binge eating.
\end{abstract}




\section{Conclusions}

The study contributes to the therapy of women with Binge Eating addictions, as it raises awareness of therapeutic-related content that could strengthen women and help them to cope with situations in their lives without the need to Binge. One of the significant variables for therapeutic work is the level of differentiation of the self. In addition, Identifying the types of defense mechanisms might help to match treatment to the woman's emotional needs. The current study found also that it is important to identify the environmental systems by which the addict is surrounded, such as whether woman is in an abusive relationship.

Finally the study leads to the recognition that Binge Eating, which is usually treated with an emphasis on nutritional behavior change, is an addiction, and as such, it requires a combination of mental, nutritional and behavioral therapy. In view of this approach it is recommended that treating a woman who is addicted to Binge Eating should involve a multidisciplinary team comprised of physicians, clinical dietitians and clinical psychotherapists.

\section{Literature Reference}

Barth, F. D. (2003). Separate but not alone: Separation-individuation issues in college students with eating disorders. Clinical Social Work Journal, 31, 139-153. Davis, C. (2013). Compulsive overeating as an addictive behavior: overlap between food addiction and binge eating disorder. Current Obesity Reports, 2, 171178.

Keywords Binge Eating Psychological Abuse 